\title{
Diagnostic Evaluation of Pulmonary Embolism During the COVID-19 Pandemic
}

\author{
Lionel S. Zuckier ${ }^{1}$, Renée M. Moadel ${ }^{1}$, Linda B. Haramati ${ }^{2}$, and Leonard M. Freeman ${ }^{1}$
}

${ }^{I}$ Division of Nuclear Medicine, Department of Radiology, Montefiore Medical Center and Albert Einstein College of Medicine, Bronx, New York; and ${ }^{2}$ Division of Thoracic Radiology, Departments of Radiology and Medicine, Montefiore Medical Center and Albert Einstein College of Medicine, Bronx, New York

$\mathbf{T}$ he rapid emergence of the COVID-19 pandemic has altered the risk-benefit calculus of many activities, including the practice of medicine. COVID-19 is caused by a single-strand RNA virus, SARS$\mathrm{CoV}-2$, belonging to the Coronaviridae family. The virus is known to infect the upper respiratory tract and can spread through aerosolization of upper airway secretions in infected patients. Indeed, current online guidance from the U.S. Centers for Disease Control and Prevention recommends that when performing aerosol-generating procedures, health-care personnel in the room should optimally wear an N95 or higher level respirator, eye protection, gloves, and a gown.

It is, therefore, crucial to identify studies that have a potential to result in aerosolized secretions and adjust to mitigate risk. The main candidate study for this intervention in nuclear medicine is ventilation/perfusion scintigraphy for the detection of pulmonary embolism. The population of patients suspected of having pulmonary embolism have overlapping symptoms with individuals who are infected with COVID-19. The typical protocol for lung scintigraphy entails performance of a low-dose ventilation examination followed immediately thereafter by a higher dose perfusion study. Previous literature has documented a small degree of radioactive contamination produced by leakage of the aerosol from the closed delivery system into the room $(1,2)$, with the potential for expired air and aerosolized secretions to contaminate personnel within the imaging suite. In addition, patients frequently cough after inhalation of a radiopharmaceutical, which may further expose nuclear medicine workers to aerosolized secretions. Although these contamination studies were performed using specific DTPA aerosol systems, various issues of leakage, aerosolization, and coughing are potentially common to all techniques of ventilation scanning, including those using radiogases, and raise concern. One potential response would be to equip technologists in the imaging suite with adequate respiratory equipment such as N-95 masks when ventilation studies are performed; however, appropriate personal protection equipment may be in short supply and not easily procured. Furthermore, after suspected aerosolization, the

Received Mar. 19, 2020; accepted Mar. 21, 2020.

For correspondence contact: Lionel S. Zuckier, Division of Nuclear Medicine, 1695A Eastchester Rd., Bronx, NY 10461.

E-mail: Izuckier@montefiore.org

Published online Apr. 1, 2020.

Immediate Open Access: Creative Commons Attribution 4.0 International License (CC BY) allows users to share and adapt with attribution, excluding materials credited to previous publications. License: https://creativecommons. org/licenses/by/4.0/. Details: http://jnm.snmjournals.org/site/misc/permission. xhtml.

COPYRIGHT @ 2020 by the Society of Nuclear Medicine and Molecular Imaging. DOI: 10.2967/jnumed.120.245571 $\gamma$-camera and imaging suite must undergo a decontamination process, significantly retarding patient throughput. We believe that under these circumstances, it is preferable to shift to a diagnostic algorithm that obviates ventilation (3-5), thereby minimizing potential for aerosolization. One such algorithm is indicated in Figure 1.

An initial step in reducing the number of ventilation scans is to rigorously assess pretest probability and limit imaging to appropriate patients. In this regard, diagnostic scoring systems such as the Wells' criteria, Pulmonary Embolism Rule-out Criteria (PERC), or the Geneva scoring system should be applied. Once a reasonable a priori probability of embolus has been established, the presence of lung parenchymal opacities should be assessed on a current chest radiograph or CT. When lung opacification is present, the

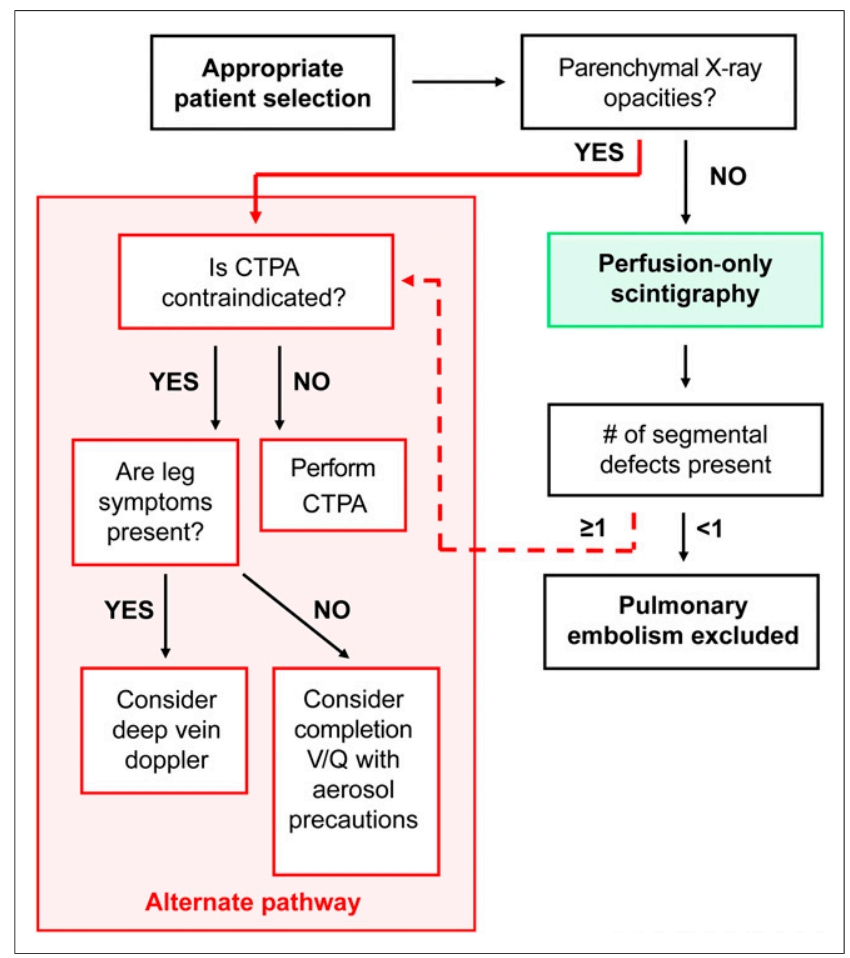

FIGURE 1. Updated diagnostic algorithm for evaluation of pulmonary embolism, which minimizes performance of ventilation studies. Green box represents perfusion scintigraphy, whereas red dotted box encloses alternate diagnostic examinations that are performed due to prior radiographic opacity (red solid arrow) or indeterminate scintigraphy (red dotted arrow). 
patient should be referred for alternate testing, generally with CT pulmonary angiography (CTPA). If the lungs are clear, we propose that the patient should proceed to perfusion scintigraphy, using either planar or tomographic imaging. If the perfusion scan does not demonstrate segmental defects, the scan is deemed negative for embolism. When segmental defects are present, the scan should be interpreted as indeterminate and the patient referred for alternate testing if greater certainty is required.

As a general rule, alternate testing should consist of CTPA. When leg symptoms are present or in the case of CTPA contraindications such as contrast allergy, performance of deep vein Doppler studies to assess for deep vein thrombosis can be considered. Alternatively, when mandated by the clinical situation, a full ventilation/ perfusion study with appropriate aerosol precautions may be completed. It is possible to perform ventilation studies on the same day as the perfusion study with a larger dosage of inhaled radiopharmaceutical if the perfusion study was performed using low levels of radiopharmaceutical. Conversely, the study can be repeated on a subsequent day.

In summary, tectonic upheavals in our clinical world necessitate a change in our approach to previously settled diagnostic algorithms; we suggest reverting to a nonventilation approach for the evaluation of pulmonary embolus to minimize potential exposure of aerosolized secretions to others in the nuclear medicine suite.

\section{DISCLOSURE}

Leonard Freeman is a member on the advisory panel of Jubilant Pharma. No other potential conflict of interest relevant to this article was reported.

\section{REFERENCES}

1. Williams DA, Carlson C, McEnerney K, Hope E, Hoh CK. Technetium-99m DTPA aerosol contamination in lung ventilation studies. J Nucl Med Technol. 1998;26:43-44.

2. Achey B, Miller K, Erdman M, King S. Potential dose to nuclear medicine technologists from ${ }^{99 \mathrm{~m}}$ Tc-DTPA aerosol lung studies. Health Phys. 2004;86:S85-S87.

3. Miniati M, Pistolesi M, Marini C, et al. Value of perfusion lung scan in the diagnosis of pulmonary embolism: results of the Prospective Investigative Study of Acute Pulmonary Embolism Diagnosis (PISA-PED). Am J Respir Crit Care Med. 1996;154:1387-1393.

4. Sostman HD, Miniati M, Gottschalk A, Matta F, Stein PD, Pistolesi M. Sensitivity and specificity of perfusion scintigraphy combined with chest radiography for acute pulmonary embolism in PIOPED II. $J$ Nucl Med. 2008;49:1741-1748.

5. Bajc M, Miniati M, Jogi J, Stein PD. Perfusion SPECT in patients with suspected pulmonary embolism. Eur J Nucl Med Mol Imaging. 2013;40:1432-1437. 